\title{
Circuit
}

Musiques contemporaines

\section{L’oeuvre de Serge Garant, ou la musique de la sensibilité secrète}

\section{The works of Serge Garant (or, the music of a secret sensibility)}

\section{Serge Provost}

Volume 7, numéro 2, 1996

Serge Garant

URI : https://id.erudit.org/iderudit/902175ar

DOI : https://doi.org/10.7202/902175ar

Aller au sommaire du numéro

Éditeur(s)

Les Presses de l'Université de Montréal

ISSN

1183-1693 (imprimé)

1488-9692 (numérique)

Découvrir la revue

Citer cet article

Provost, S. (1996). L'oeuvre de Serge Garant, ou la musique de la sensibilité secrète. Circuit, 7(2), 15-22. https://doi.org/10.7202/902175ar
Résumé de l'article

L'auteur propose une réécoute de son œuvre en insistant sur l'équilibre, chez Garant, entre les exigences techniques et la sensibilité. Il propose un parcours chronologique de son catalogue en soulignant les grandes caractéristiques de chacune des phases de son activité créatrice. 


\section{VISAGES}

\section{L'œuvre de Serge Garant, ou la musique de la sensibilité secrète Serge Provost}

C'est une expérience assez singulière que de réécouter sur disques l'ensemble de l'œuvre de Serge Garant. On a l'impression de redécouvrir une musique que, finalement, on connaissait mal ou trop peu, et surtout de découvrir à travers elle des traits de la personnalité profonde de son auteur. II est étonnant de constater qu'avec le temps, la perception d'une œuvre d'art peut évolver et même changer de façon assez radicale, comme s'il s'agissait d'une révélation. La musique de Garant apparaît aujourd'hui, dans son ensemble, d'une fraîcheur et d'une force étonnante, mais surtout d'une clarté décapante qui la distingue. Pourtant, dès les premières notes, on a le réflexe de classer, de dater; la musique de Garant appartient en effet à une époque qui glisse irréversiblement vers hier. Mais cette distance nous permet de voir tout à coup les choses autrement, d'écouter sans parti-pris. Le plus frappant, c'est qu'une musique réputée difficile, intellectuelle, une musique dans laquelle toute référence au "moi ", au psychodrame autobiographique a été consciemment exclue par son auteur, nous livre à ce point une sensibilité vibrante, raffinée et un tempérament vigoureux.

Pour moi, l'émotion existe mais i'ai horreur d'en parler ; c'est fondamental, bien sûr, sinon une œuvre ne respire pas. Seulement le compositeur ne doit pas s'en soucier vraiment. [...] La partie la plus intéressante d'un être, c'est toujours ce qu'il y a de plus secret, de plus profond. L'émotion doit naître de cette magie mystérieuse qui se produit quand les problèmes techniques sont si bien résolus qu'on ne peut imaginer qu'il y en ait eu'"I. (Garant, 1978 in Lefebvre, 1986, p. 183.)

\section{Question de choix}

Dans les années qui ont suivi la fin de la dernière guerre mondiale, les jeunes artistes québécois ont ressenti la nécessité de s'ouvrir au monde, de participer
(1) Entrevue avec Serge Garant, reprise dans l'Anthologie de la musique canadienne, coffret $\mathrm{RCl}$, vol. 1-4, RadioCanada International, 1978. Sauf précision contraire, tous les propos de Garant cités dans cet article sont extraits du livre de Marie-Thérèse Levebvre 11986). 
aux grands mouvements intellectuels, artistiques, sociaux de leur époque, et aussi de se définir, de se trouver une identité propre selon la nature et les aspirations de chacun. Au retour d'un séjour d'un an à Paris, Garant hésite entre deux mondes : une écriture polymodale inspirée de Messiaen et la technique sérielle. Mais, à partir de 1954, les choix de Garant sont clairs et délibérés, marqués par le refus de la tradition, non seulement dans le cadre québécois, où ce refus a été le fait de toute une génération d'artistes ${ }^{(2)}$, mais dans un refus général de la tradition musicale occidentale len fait, de tout l'héritage du XIX siècle). Garant se lance dans l'aventure sérielle, emboîtant le pas en quelque sorte à Pierre Boulez let cette communauté de pensée entre les deux compositeurs a été assez évidente durant toutes les années cinquante). La découverte de la musique de l'école de Vienne, de celle de Webern en particulier, a cristallisé cette volonté de créer un nouveau monde sonore, un nouvel ordre musical, en quelque sorte. Ainsi, très tôt, Garant s'est positionné très clairement et sa première période de production, des Caprices (1954) à Asymétries $n^{\circ} 2$ (1959), en est le strict reflet.

Les motivations d'un tel choix sont de deux ordres. Le premier, comme on l'a dit, fondé sur le refus et même sur le rejet de ce qui a précédé. Si l'on se replace dans le contexte de l'époque, ce genre d'attitude, est facile à comprendre. Au lendemain d'un grand cataclysme, en l'occurrence la guerre, c'est tout un monde qu'on rejette, un monde où tout semble corrompu ou marqué par la compromission, décadent et obsolète, la musique y compris. Les réactions viscérales, violentes du jeune Boulez, par exemple, sont à cet égard assez éloquentes.

Dans cette mouvance, la musique d'Anton Webern est apparue comme une réponse éclatante à l'essoufflement post-romantique et à la sécheresse étriquée du néo-classicisme, comme un refus de tous les conservatismes et, pour les jeunes compositeurs italiens (Maderna, Nono), un symbole de la résistance antifasciste. Dans le contexte québécois de l'époque, c'est plutôt la lourdeur étouffante d'un modèle de société archaïque qui est mis en cause, et le sentiment de révolte des jeunes Européens a trouvé dans le jeune Garant un écho fraternel.

Mais au-delà de ces considérations socio-politiques, c'est l'authentique fascination exercée par l'art de Webern qui a mis Garant en face de ses propres aspirations et d'un deuxième niveau de motivation, plus profond ef plus intime. Le compositeur a ressenti le besoin de se trouver en terrain vierge, de se forger des outils neufs, de se créer un langage libéré de charges historiques, émotionnelles et esthétiques, afin d'y couler sa pensée, sa sensibilité, avec le plus de cohérence, rigueur... et de détachement - du moins apparent possible. En fait, une recherche de pureté - j'oserais dire d'une nouvelle virginité de l'art -, une sorte d'idéalisme aussi, pousse Garant à faire une musique à l'image d'un monde neuf purifié de ses vieux démons. Serait-ce une façon
(2) En particulier le groupe des automatistes, dont l'inspirateur était PaulÉmile Borduas. 
d'occulter ses angoisses existentielles ? Peut-être, mais aussi le besoin légitime d'explorer de nouveaux mondes, à une époque où l'on croyait que ceux-ci pouvaient encore exister. Les positions de Garant, en ce qui a trait à sa propre création, se sont toutefois assouplies et sa démarche a été marquée par une constante, mais lucide recherche de liberté, davantage que par un besoin d'orthodoxie. Une pensée musicale fondée au départ sur l'exclusion s'est peu à peu ouverte à l'intégration.

Cet art lumineux [celui de Webern], équilibré, suprêmement intelligent, passe encore mal la rampe à cause de sa perfection même. Tant de discrétion, de finesse, de pureté laisse assez peu filtrer l'importance d'une démarche dont la musique portera à jamais la marque incandescente. (Garant, 1969 in Lefebvre, 1986, p. 154.)

\section{Itinéraire}

La production musicale de Garant comple cinquante-cinq œuvres répertoriées (dont des musiques de scène et quelques pièces de moindre importance), conçues en trente-cinq ans d'écriture, soit de 1946 (Conte pour piano) à 1981 (Plages pour orchestre). Son itinéraire de création nous semble avoir toujours évolué autour de l'axe " rigueur-liberté » dans un mouvement de l'une vers l'autre marqué par une volonté d'intégration de plus en plus grande de nouveaux éléments - techniques d'écritures, modes d'expression, conceptions esthétiques - et ce tout en restant fidèle à un constant souci de cohérence et de clarté. Comme chez la plupart des créateurs, cette évolution s'est faite par étapes, ou périodes, dont les œuvres représentent les jalons.

\section{Rigueur I}

La première période, plus particulièrement de 1954 à 1959, est consacrée à l'acquisition des techniques sérielles et à la constitution d'un vocabulaire de base. Nous retiendrons comme exemples Nucléogamme, Asymétries $n^{\circ} 1$ et Asymétries $n^{\circ} 2$. Ces trois pièces montrent bien l'évolution du compositeur pendant cette courte période. Dans la première, Nucléogamme, pour ensemble à vent, piano et bande magnétique, la distribution du matériau sériel (les six premiers sons de la série do l'opus 21 de Webern) est encore assez mécanique et confère une certaine raideur au discours, raideur qui est toutefois contrebalancée par la bande magnétique dont l'emploi assez ingénieux capte l'attention. Dans Asymétries $n^{\circ} 1$, pour piano, le geste musical est beaucoup plus marqué, le matériau plus caractérisé et aussi plus concentré. II y a dans cette pièce une âpreté qui va devenir une marque de style chez Garant. Mais c'est dans Asymétries $n^{\circ} 2$, pour clarinette et piano, que le compositeur 
atteint une véritable maîtrise de son langage de base. L'écriture est beaucoup plus déliée; la forme, plus libre et mieux découpée, intègre par endroit des éléments aléatoires (le mouvement $B$ ).

\section{Liberté I}

Anerca, pour voix de soprano et ensemble, composé en 1961, représente un point tournant dans la production de Serge Garant et inaugure toute une période d'expérimentations qui va libérer le compositeur de l'orthodoxie postsérielle et l'amener à des conceptions beaucoup plus ouvertes du phénomène musical. Garant conquiert ainsi de nouveaux territoires. Le traitement du temps et de la forme, échappant à des stuctures entièrement prédéterminées, suscite un geste musical beaucoup plus spontané. Le traitement plus conscient du timbre et du rythme, l'intégration de nouveaux modes de jeu, l'établissement de plans sonores amènent des éléments de contraste qui donnent à sa musique une dimension vivante et crée pour ainsi dire une "dramaturgie " du son. $\grave{A}$ l'obsession de la cohérence et de la clarté à tout prix s'ajoute maintenant l'inquiétude fondamentale du temps et de l'espace. Ces conceptions viennent d'une part du choc causé par certaines œuvres de Stockhausen, telles Zeitmasse, Gruppen et le Klavierstück XI. La notion de "hasard contrôlé " a particulièrement marqué Garant et trouvera une application féconde dans les œuvres qui vont suivre.

La solution proposée par Stockhausen n'est pas unique, et on reste rêveur devant les possibilités que nous offrent les " hasards contrôlés » appliqués à un ensemble instrumental. On imagine assez la mobilité, la fantaisie, le caractère unique d'une œuvre spatiale réalisée par cinq, dix ou cinquante interprètes... (Garant, Le Journal musical canadien, février 1959 ; cité in Lefebvre 1986, p. 132.)

Il faut dire que la rencontre du peintre québécois Fernand Toupin en 1960 va donner une profondeur à ces concepts et « moduler » la pensée de Garant de façon à lui donner encore plus de force et d'originalité.

Je suis absolument passionné par la mise en jeu d'une certaine quantité "d'événements musicaux » qui ne sont pas donnés comme fixes dans leur état ni dans leur ordre d'apparition. Je ne compose qu'un schéma de base, une structure mobile qui donne, à l'exécution, une série d'improvisations dirigées. Personnellement je ne crois plus au langage discursif, à l'œuvre qui commence et qui finit. Je cherche une temporalité ouverte. Jusqu'en 1959, les structures que je créais étaient totalement organisées. Depuis je travaille par "matière", comme un peintre; c'est du reste la peinture qui m'a amené à cette conception: remplir des espaces de 
matière, pour moi un espace-temps et une matière sonore, sans égard pour une conception préétablie. (Garant, Culture vivante, n 5, 1967 ; cité in Lefebvre, 1986, p. 117.)

De cette période d'expérimentation qui va de 1961 à 1969, on retiendra surtout le tryptique constitué de Phrases / 1 1967, mezzo-soprano, piano, percussions, célesta), Phrases II (1968, deux orchestres) et Jeu à quatre (1968, quatre groupes instrumentaux). Ces trois pièces forment une espèce de boucle. Dans la première, le dualisme rigueur-liberté est clairement posé ; le système de proportions, le rapport entre les éléments structurels et la grande forme sont strictement déterminés, alors que le parcours de l'œuvre /l'agencement en temps réel de ses composantes) demeure ouvert.

Phrases /l va encore plus loin dans ce sens. L'organisation de séquences se fait à partir de modules de base; à une cellule intervallique (le demi-ton) correspond une durée de base (1 4 ou 16 secondes); enfin, un système de proportions (le rapport $7 / 8$ ) règle toute l'organisation. Est ainsi générée une double version de la pièce, laquelle doit être exécutée simultanément par deux orchestres dont les trajets sont déterminés par les chefs respectifs (le résultat perçu par le public est une troisième version, addition des deux premières). L'utilisation de la voix des instrumentistes comme élément matériel, "bruitiste ", donne ici une dimension sonore - mais aussi conceptuelle - intéressante, évoquant Luigi Nono par l'intégration de textes à portée politique (Pierre Bourgault et "Che » Guevara, respectivement pour Phrases / et III.

C'est une musique REVENDICATRICE. [...] Et pourquoi le musicien ne pourrait-il pas témoigner dans ces œuvres comme un écrivain par exemple ${ }^{(3)}$.

Jeu à quatre (1968) referme la boucle en ce sens que la mobilité du matériau des deux premières pièces (les trois pièces étant construites sur les mêmes éléments de base) redevient ici directionnelle et entièrement contrôlée par l'écriture.

\section{Intégration}

Les deux grands cycles Offrandes I, II et III ; Circuits I, II et III (1969-1973) constituent en quelque sorte la clé de voûte de l'œuvre de Garant. Ils intègrent tous les acquis de la période précédente : liberté d'invention, vocabulaire riche et varié, explorations formelles, travail de la matière sonore. De plus, la notion de modèle est ici réinventée, puisque ces œuvres découlent toutes du thème de l'Offrande musicale de J. S. Bach, utilisé non pas comme citation ou élément de collage (sauf occasionnellement dans Offrande I) mais comme matériau générant un système de proportions qui sera le moteur des six pièces et
(3) " "La musique moderne c'est du caviar." Entrevue avec Jacques Thériault », Le Devoir, 6 avril 1968. 
qui touchera tous les aspects de la composition : timbres, dynamiques, temps, filtres, réseaux harmoniques, etc. L'intégration du modèle sera poussée à ses ultimes limites, le thème célèbre étant de plus en "absorbé " d'une pièce à l'autre jusqu'à n'être plus qu'un système abstrait de proportions numériques (telle la série de Fibonaccil qui servira de canevas au jeu des percussionnistes dans Circuit II et se résorbera autour d'un son pivot (fa dièse) et de plages de temps dans Circuit III, la pièce la plus abstraite du groupe.

Cette période, caractérisée par une instrospection grandissante, marque une sorte d'aboutissement et peut-être même la limite atteinte dans la voie de l'abstration et de la concentration de l'idée musicale. Quoi qu'il en soit, ces œuvres sont d'une solidité et d'une profondeur de pensée tout à fait remarquables.

\section{Liberté II}

Après une période de doutes et de réflexions marquée en partie par la rencontre de Claude Vivier, le génie de Serge Garant éclate dans une œuvre surprenante, ... chant d'amours (1975). Le lyrisme puissant, l'immédiateté du geste, la teneur du contenu, le choix des textes - sorte de "Traité de l'amour " sous toutes ses formes -, la volonté de communiquer directement le contenu sémantique sans l'atomiser dans des structures abstraites, tout cela témoigne d'une liberté créatrice extraordinaire et assumée ainsi que d'un geste humain assez remarquable pour l'artiste qui a toujours occulté tout élément « personnel » de sa création. ... chant d'amours est un long cri modulé de désirs et de passion, un geste de libération.

\section{Rigueur II (poésie)}

Après Rivages (1976, baryton et orchestre), œuvre d'allure assez dramatique et nettement plus sombre sur un texte d'Alain Grandbois, l'année 1978 voit apparaître Quintette, qui témoigne d'un retour à la concentration, à l'instrospection, à la rigueur. Cette partition admirable témoigne d'une maturité, d'une élévation et surtout d'une poésie pénétrantes. L'écriture expressive et fluide s'est dégagée de l'aspect anguleux des œuvres plus anciennes, ef livre un discours musical profondément émouvant qui ressemble à un testament.

\section{Postlude en forme d'adieu}

Plages, de 1981, commande de l'Orchestre des jeunes du Québec, est la dernière œuvre de Serge Garant. Écrite dans un langage renouant avec 
I'harmonie traditionnelle, c'est une œuvre sombre aux accents tragiques (le début évoque Mahler). On ne peut manquer d'y entendre l'expression d'une forme de désarroi, d'une crise intérieure. Garant disait de cette œuvre qu'elle resterait, dans sa production, une parenthèse. Parenthèse qui ne s'est jamais refermée.

Le silence des dernières années témoigne de cet étal de crise. Le compositeur se sentaitil isolé par l'évolution du goût musical ? Fautil y voir une impossibilité de s'adapter, de se renouveler (comme l'ont fait Berio et ligeti, par exemple) ? S'agissaitil d'une crise plus intime? la question demeure. Je me risquerai à dire simplement que dans un monde musical où il ne se reconnaissait plus, Garant a préféré se taire par fidélité à soi-même.

\section{Héritage}

La musique de Serge Garant est aujourd'hui méconnue, non seulement du public, mais aussi des musiciens et même des jeunes compositeurs. Pourtant, il s'agit d'une œuvre très riche d'enseignements de tous ordres. On peut surtout retenir cette dialectique entre la pensée spéculative et le contact avec la mafière et constater à quel point ce dosage d'autocritique et d'expérimentation a été fécond. Enfin, il est extrêmement regrettable qu'un véritable trésor collectif soit ainsi ignoré, comme l'est en général la création musicale en notre pays. Parmi les créateurs, le compositeur d'ici est le moins considéré - «béluga culturel ", comme a déjà dit Gilles Tremblay -, quand il n'est pas carrément méprisé ou insulté, et ce, dans les milieux culturels même. Toute sa vie durant, Serge Garant nous a donné le meilleur de lui-même; il a été et demeure par son œuvre un artiste exemplaire. Sa musique restera un apport essentiel à la construction de notre culture et surtout, humainement, le témoignage d'un authentique créateur.

On n'a pas le droit de s'en priver et d'en priver les autres. Il est plus que temps de soulever le voile du silence qui pèse sur sa musique, sur notre musique. Peut-être peut-on résumer ainsi le message ultime de Serge Garant : la conquête incessante d'une plus grande LIBERTE.

GARANT, S. (1969), Programme de la SMCQ, no 19, 28 octobre.

LEFEBVRE, M.Th. (1986), Serge Garant et la révolution musicale au Québec, Montréal, Lovise Courteau éditrice. 


$$
\text { يدي }
$$

